\title{
ENSINO REMOTO DE TECNOLOGIA DA INFORMAÇÃO APLICADA À AUTOMAÇÃO ATRAVÉS DE UMA PLATAFORMA INDUSTRIAL DE DADOS
}

DOI: $10.37702 / 2175-957 X . C O B E N G E .2021 .3572$

Marco Aurelio de Souza Birchal - birchal@pucminas.br

PUC Minas Pontifícia Universidade Católica de Minas Gerais

rua padre pedro evangelista 274

30535-490 - Belo Horizonte - MG

JÚLIO CESAR BRAZ DE QUEIROZ - jqueiroz@pucminas.br

PUCMG

Rua das Hortênsias 262

34010-579 - Nova Lima - MG

Resumo: Sistemas PIMS (Plant Information Management Systems) são parte importante do aprendizado de tecnologias de informação aplicadas à engenharia de controle e automação. $O$ estudo dessas tecnologias é potencializado através de aulas práticas de laboratório. $O$ advento da pandemia tornou impossível o acesso físico do aluno aos laboratórios. Este trabalho demonstra uma solução tecnológica de ensino à distância, através do emprego de VPN (Virtual Private Network) para acesso à infraestrutura de rede. Trata-se também das principais funcionalidades de um sistema PIMS que devem ser dominadas por um engenheiro de controle e automação.

Palavras-chave: PIMS. Ensino Remoto. Tecnologia da Informação Aplicada. 


\section{(C) COBENGE \\ ENSINO REMOTO DE TECNOLOGIA DA INFORMAÇÃO APLICADA À AUTOMAÇÃO ATRAVÉS DE UMA PLATAFORMA INDUSTRIAL DE DADOS}

\section{INTRODUÇÃO}

O cursos de Engenharia de Controle e Automação, assim como outros, afins, frequentemente possuem disciplinas que tratam do emprego ou da integração de sistemas de tecnologia da informação às tecnologias de automação de sistemas em seus Projetos Pedagógicos.

A pirâmide da automação, estabelecida pela ISA 95 (ISA 2010), ilustrada na Figura 1 e tão recorrentemente citada e estudada quando se trata de se explorar as fases ou interações dos dados em um sistema de automação, prevê o inter-relacionamento das tecnologias de informação com os sistemas de manufatura automatizada.

Figura 1 - A Pirâmide da automação segundo a ISA-95.

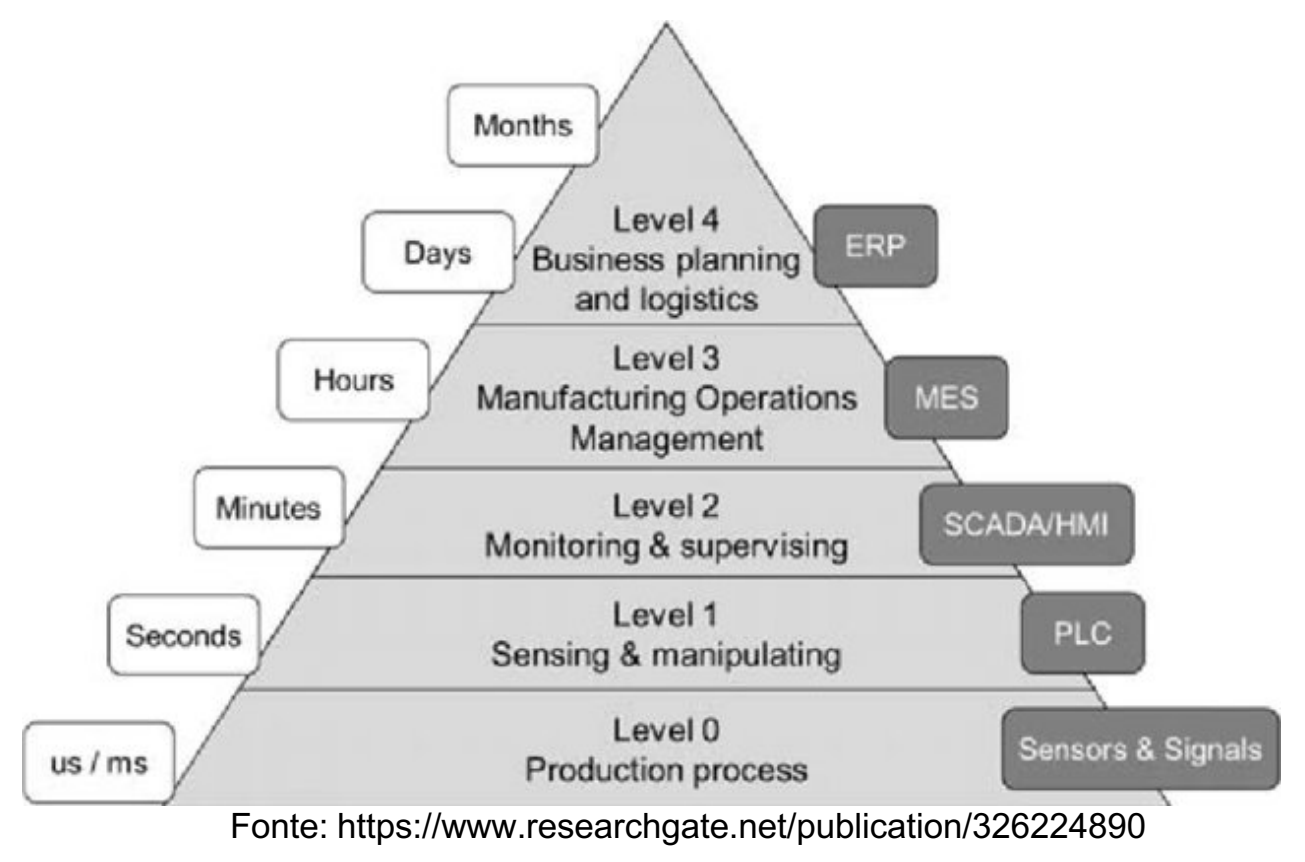

Os níveis 0 a 2 são essencialmente níveis clássicos de tecnologia da automação. $A$ partir do nível 3, veem-se as tecnologias de informação que, num primeiro momento, não participavam do escopo de matérias da engenharia, mas sim, dos cursos de ciência da computação e suas vertentes. A realidade atual, no entanto, aproxima o engenheiro contemporâneo das camadas superiores da pirâmide, fazendo com que esses temas, essencialmente computacionais, passem a fazer parte do arcabouço de competências necessárias a este novo engenheiro.

Com o advento da pandemia de COVID/19, no entanto, o desafio do ensino desta matéria se tornou ainda maior, uma vez que sistemas de extração e tratamento de dados industriais, são, muitas vezes, sistemas complexos que devem estar instalados em uma infraestrutura de rede local, à qual o usuário deve ter acesso para que possa utilizá-los.

Este trabalho apresenta a disciplina Laboratório de Tecnologia da Informação Aplicada à Automação, do currículo atual do curso de Engenharia de Controle e Automação 
da PUC Minas, bem como a solução para o ensino remoto da mesma, através do emprego de acesso remoto via VPN (Virtual Private Network) (KUROSE \& ROSS, 2013).

\section{AULAS PRÁTICAS DE TI APLICADA}

A camada 3 da pirâmide da automação é a camada dos sistemas MOMs (Manufacturing Operation Management), da qual participam, além do MES (Manufacturing Operations Management), muitos outros sistemas, como o LIMS (Iaboratory information management system) e o PIMS (Plant Information Management Systems).

A disciplina em questão, é uma disciplina prática e semanal, que ocorre em um laboratório de automação, em que os alunos tem acesso ao PI System, da OsiSoft. Para que se conduza de forma fluida, há três momentos distintos: uma introdução à disciplina que apresenta o sistema a ser utilizado; um primeiro momento de contato do aluno com o software, em que há apresentação de um estudo de caso prático e o desenvolvimento de um projeto, em grupo. A seguir, cada uma dessas etapas é detalhada.

\subsection{Introdução ao tema e ao software}

Inicialmente, o aluno é apresentado à questão das tecnologias de informação que dão suporte à automação e da interação entre elas. Apresentam-se, portanto, as várias ferramentas de nível 3. Em seguida, o PIMS é apresentado e, como representante desta tecnologia, o PI System e seus elementos.

O PI System é um software de PIMS que é uma plataforma de gerenciamento para coleta os dados em tempo real, armazenamento e tratamento para posterior visualização e entrega dos dados de forma conveniente ao usuário (OsiSoft, 2018).

Sendo um sistema complexo e com muitos elementos, há necessidade de exploração de cada um deles para uq se possa compreender seu funcionamento. Essencialmente, o PI System é um sistema cliente-servidor, que roda em uma estrutura distribuída. A Figura 2 ilustra os principais componentes desse sistema.

No caso específico que está sendo tratado, O servidor - PI Server - fica instalado junto à infraestrutura de TI da universidade e é acessado através da rede local. Os alunos utilizam de clientes - Pl visualization Tool - instalados nos computadores do laboratório para acesso ao servidor. Nesta etapa, os alunos são apresentados esta estrutura e tem o primeiro contato com a mesma, de forma a contextualizar o ambiente de trabalho e para que se familiarizem com o mesmo.

Figura 2 - Componentes do PI System.

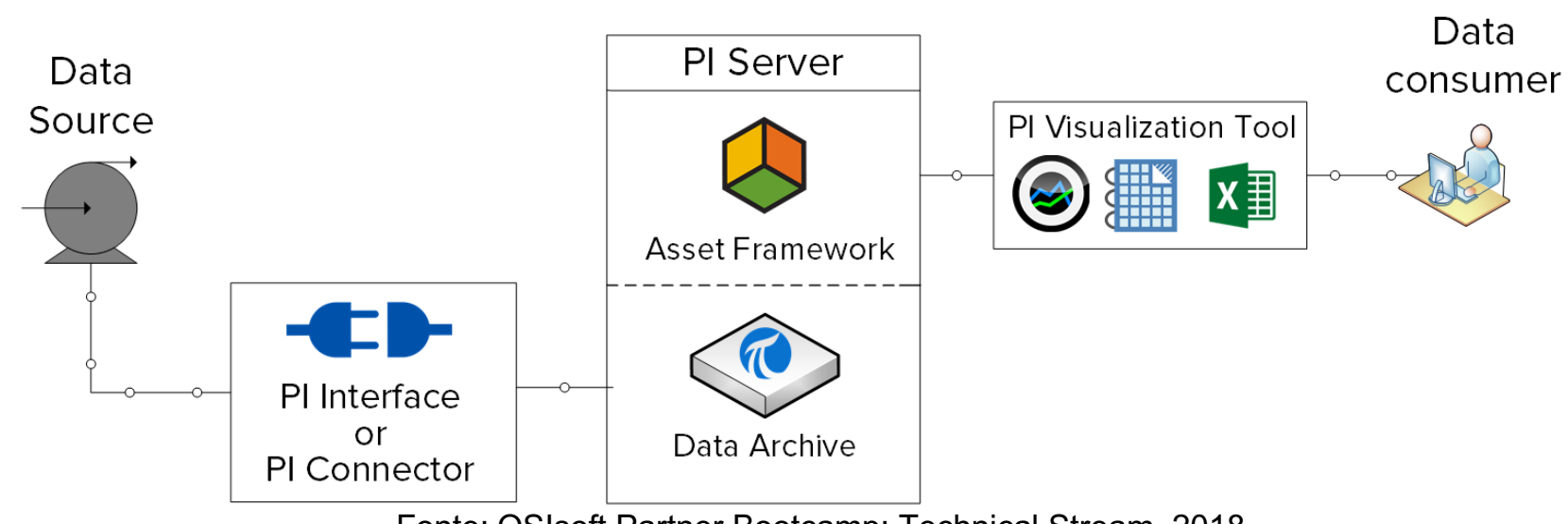

Fonte: OSIsoft Partner Bootcamp: Technical Stream. 2018. 


\section{$2.2 \quad$ Estudo de caso}

No segundo momento da disciplina, faz-se um estudo de caso de um sistema real, que controla as perdas por vazamento de um sistema de abastecimento de água. O problema é apresentado e o aluno é levado a reproduzir o sistema original.

Aqui, o aluno precisa operar o software de forma realística e em tempo real, criando as entidades necessárias à correta representação dos ativos do sistema a ser implementado, dentro do PI system.

Ao final deste processo, cada aluno deverá possuir, em sua conta, no PI System, uma implementação própria do estudo de caso apresentado.

\subsection{Desenvolvimento de um projeto}

$\mathrm{Na}$ terceira etapa da disciplina, que a a etapa final e mais instigante, formam-se grupos de alunos para que, cada grupo proponha e implemente um projeto próprio, que deve ser apresentado no final do semestre.

Os alunos tem a oportunidade de buscar um problema prático e implementar a solução de PIMS que o atenda. Para tanto, devem passar por todas etapas de projeto e implementação de uma solução de TI aplicada à automação.

Este é um momento de bastante criatividade, em que se veem as mais variadas representações de processos industriais, o que faz com que cada grupo possa buscar, dentro do universo da engenharia, aquilo que mais lhes é afim ou de interesse naquele momento.

No fechamento desta fase, cada grupo apresenta não só o problema escolhido, como também a solução implementada no PI System.

\section{O DESAFIO DO ENSINO REMOTO}

Todo o processo descrito no item 2 se refere às aulas presenciais, que ocorrem em laboratório da universidade, preparado para a execução do PI System e sob a supervisão de um professor responsável pela disciplina, devidamente assistido por uma equipe de $\mathrm{TI}$ de suporte à informática.

Com advento da pandemia de Covid/19, houve a necessidade de implantação do ensino remoto e a impossibilidade de acesso à infraestrutura de laboratório. Isso provocou grave crise na execução da disciplina, de caráter essencialmente prático e dependente da infraestrutura em questão.

O PI System, por sua natureza complexa, possui vários módulos que devem ser acessados pelos alunos durante a aula. Esses módulos, por sua vez, possuem sistema de autenticação de segurança tanto de usuário quanto de localidade física, o que impede ou dificulta, sobremaneira, a instalação desses módulos das máquinas pessoais dos alunos.

A solução que se apresentou foi a instalação de um acesso remoto do aluno à máquina do laboratório, que ele utilizaria se estivesse presencialmente no mesmo.

Desta forma, as aulas são conduzidas, no ensino remoto, através do programa Microsoft Teams, que cria uma sala virtual e, adicionalmente, cada aluno e o professor, logam remotamente, via VPN, no sistema da universidade e, posteriormente, em cada uma das máquinas do laboratório.

Isto deve ser feito em etapas, as quais serão apresentadas a seguir.

\subsection{Estabelecimento de uma conexão VPN}

Para que o aluno possa acessar a rede da universidade, é preciso que, do lado da instituição, haja um servidor de acesso capaz de autenticar cada aluno, reconhecendo-o como usuário válido e permitindo que ele possa acessar os recursos internos da mesma. 
Isso é alcançado utilizando-se o software Global Protect da Palo Alto Networks, por parte da instituição de ensino.

Já do lado do aluno, basta que o mesmo instale um agente de conexão com a VPN da universidade. A Figura 3 ilustra o procedimento de conexão de um aluno com a rede local da instituição via VPN.

Figura 3 - Conexão via VPN.

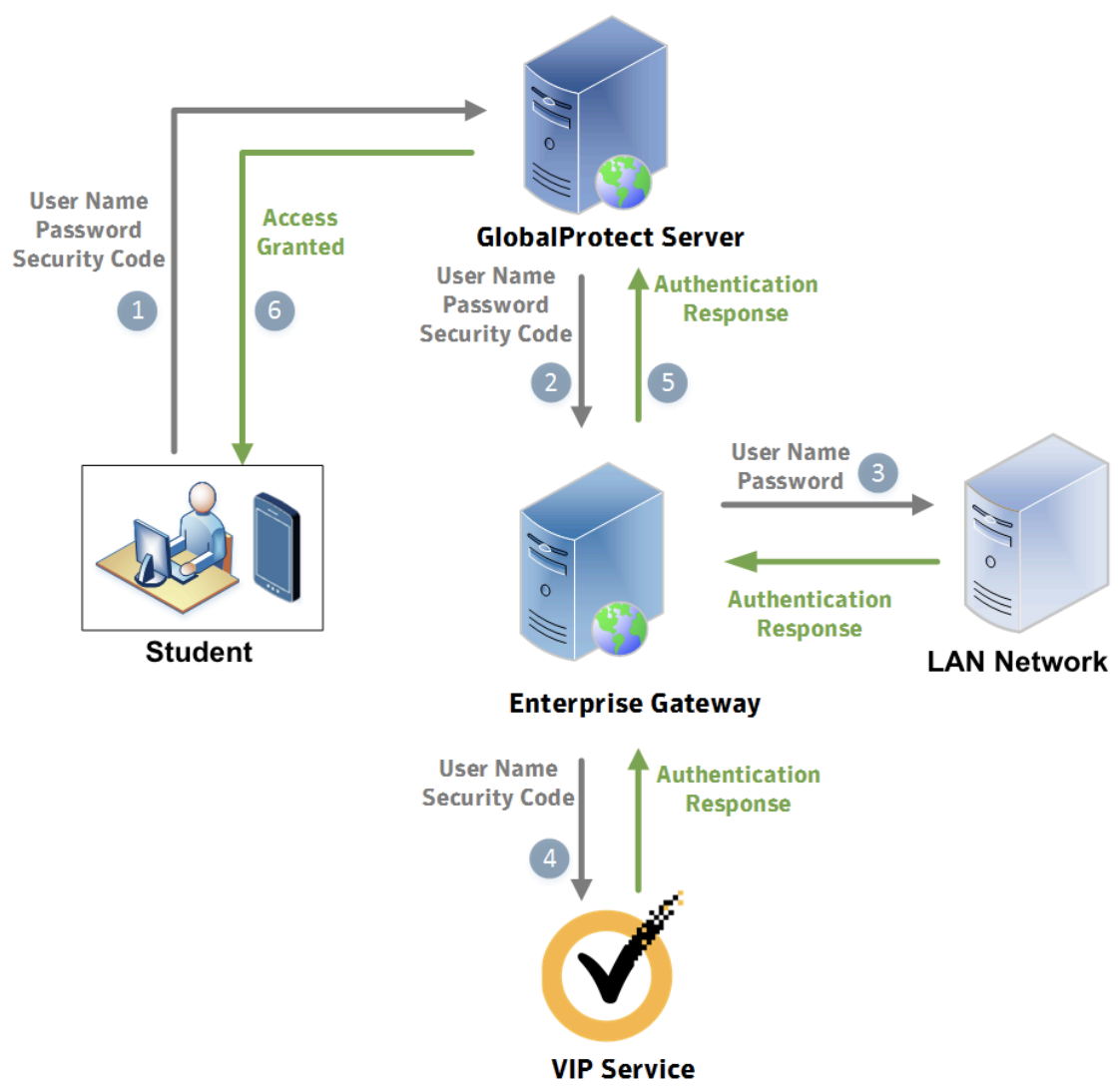

Fonte: OSIsoft Partner Bootcamp: Technical Stream. 2018.

Após a autenticação do aluno, ele passa a estar, virtualmente, conectado à rede local da instituição. Dá a designação de VPN, isto é, rede privada virtual. Uma vez, conectado à rede, o aluno deve escolher a máquina a qual ele deseja se conectar. Este momento se assemelha, no ambiente físico, a se dirigir até o laboratório e logar na máquina local, que possui todo o conjunto de programas clientes de acesso ao sistema PI System. Para que isso seja feito, utiliza-se o programa Microsoft Remote Desktop, que já se encontra instalado em todas as máquinas Windows e que permite a conexão remota as maquinas que estejam devidamente configuradas para tanto, como é o caso dos computadores dos laboratórios da instituição. A Figura 4 ilustra a conexão, por parte do aluno, tanto em uma máquina Windows como em uma máquina Apple. 


\section{COBENGE de Educação em Engenharia $2021 \mid \begin{aligned} & \text { de Educaçäo } \\ & \text { da } A B E N G E\end{aligned}$ 28 a 30 de SETEMBRO \\ Evento Online \\ "Formaçāo em Engenharia: \\ Tecnologia, Inovaçāo e Sustentabilidade"}

Figura 4 - Conexão Remota por parte do cliente.

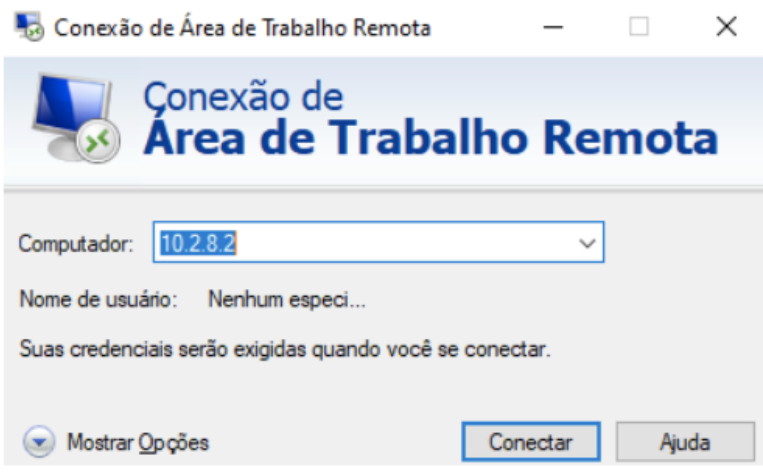

(a) Windows

Fonte: $\mathrm{O}$ autor

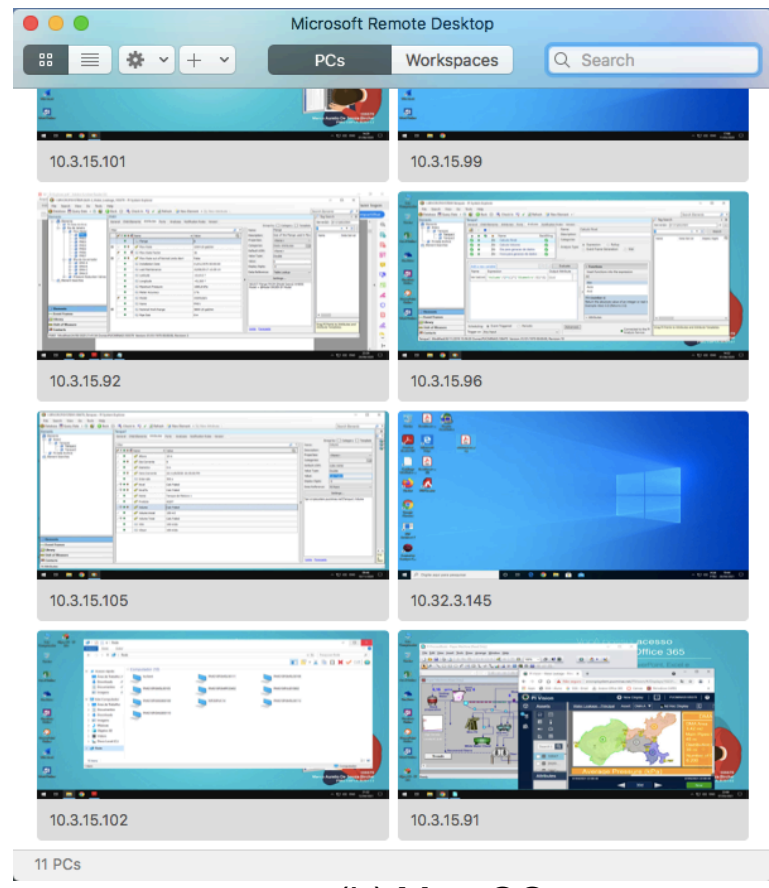

(b) Mac OS

Note-se que é necessário que a máquina a ser acessada esteja realmente ligada, no momento da conexão. Uma conectado, o aluno passa a ter, na tela do seu computador, um espelho da tela do computador do laboratório e o controle da mesma. Uma vez conectado ao computador destino, o acesso se assemelha ao apresentado pela Figura 5, que que se pode perceber o número de endereço de rede do computador acessado, na barra de título da janela demonstrada.

Figura 5 - Exemplo de acesso ao computador remoto.

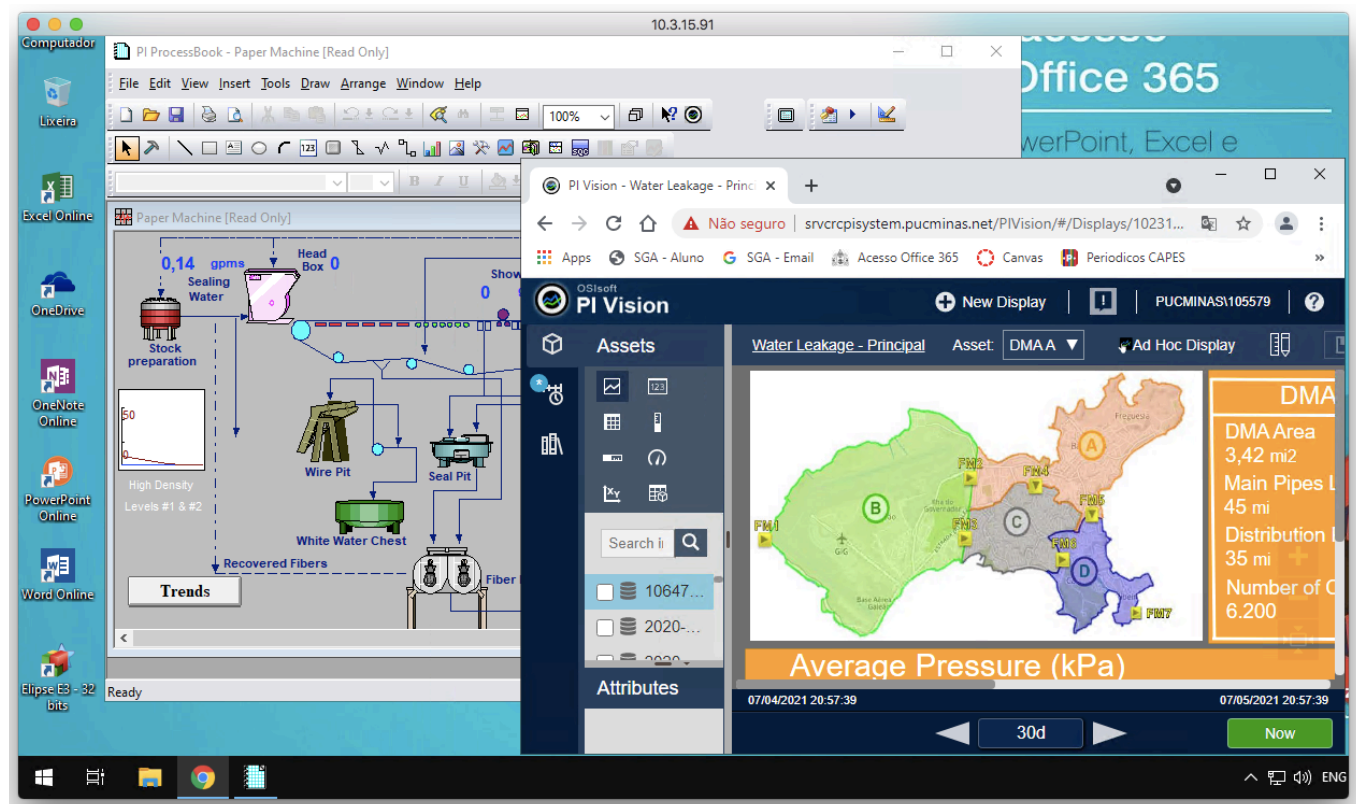

Fonte: $\mathrm{O}$ autor 


\section{CONCLUSÃO}

A necessidade do ensino de tecnologias de informação para alunos de engenharia e não somente aquelas disciplinas que classicamente compõem o conjunto de aprendizado do engenheiro, demonstra a evolução da própria engenharia e de sua interlocução com as novas tecnologias, que, até pouco tempo, eram então e somente prestadoras de serviços que pouco interessavam ao engenheiro.

O desenvolvimento de uma metodologia prática para ensino de tecnologia da informação, que permita ao aluno um contato real com softwares de produção que são encontrados no mercado e que atualmente tem sido cada vez mais empregados pelos engenheiros em seus projetos de integração do processo produtivo, fornece os meios para que o aluno se introduza, de forma consistente nesta realidade.

Trata-se de um momento intensamente multidisciplinar, que desafia cada aluno a trabalhar em sintonia dentro de seu grupo, na solução de um problema prático. Aqui exploram-se e desenvolvem-se competências as mais variadas, o que se espera e se exige da formação de um engenheiro contemporâneo.

Com o advento da pandemia, no entanto, viu-se uma barreira fática ao desenvolvimento de uma disciplina como esta. No entanto, as mesmas tecnologias da informação de se pretende ensinar ao engenheiro, foram utilizadas como ferramenta de viabilização deste ensino, o que permitiu o desenvolvimento do trabalho, antes em laboratório, a níveis equivalentes de satisfação.

Percebe-se que o produto final da disciplina, qual seja, os projetos desenvolvidos pelos grupos, tem qualidade semelhante daqueles pré-pandemia, o que demonstra a efetividade dos meios remotos no auxilio à continuidade do ensino em tempos pandêmicos.

Não se espera que este modelo perdure como está, assim como se espera que a pandemia termine o quanto antes e se volte ao novo normal tão dito por todos. Que as experiências bem sucedidas de ensino remoto possam trazer novas formas de promover o ensino e sejam ferramentas de apoio à educação.

\section{REFERÊNCIAS}

INTERNATIONAL SOCIETY OF AUTOMATION. ISA-95.00.01-2010 Enterprise-Control System - Integration Part 1: Models and Terminology. 2010.

KUROSE, James F.; ROSS, Kith W. Redes de Computadores e a Internet. 6. ed, São Paulo: Pearson Education do Brasil. 2013.

Ministério da Educação. Resolução CNE/CES n 2, de 24 de abril de 2019, Diretrizes Curriculares Nacionais do Curso de Graduação em Engenharia.

Disponível em: <http://portal.mec.gov.br/escola-de-gestores-da-educacao-basica/323secretarias-112877938/orgaos-vinculados-82187207/12991-diretrizes-curriculares-cursosde-graduacao>

OsiSoft. OSIsoft Partner Bootcamp: Technical Stream. San Leandro: OsiSoft LCC, 2018. 
SEIXAS F., C. PIMS - Process Information Management System - Uma introdução. Belo Horizonte: Editora UFMG. 2003.

\title{
USE OF AN INDUSTRIAL DATA PLATFORM AS A TOOL TO THE REMOTE ENGINEERING EDUCATION
}

\begin{abstract}
PIMS (Plant Information Management Systems) systems are an important part of learning information technologies applied to control and automation engineering. The study of these technologies is enhanced through practical laboratory classes. The advent of the pandemic made it impossible for students to physically access laboratories. This work demonstrates a technological solution for remote learning, through the use of VPN (Virtual Private Network) to access the network infrastructure. It also presents the main functionalities of a PIMS system that must be mastered by a control and automation engineer.
\end{abstract}

Keywords: PIMS. Remote Learning. Applied Information Technology 